$2^{4}$

ORIGENES Y PROBLEMAS TEORICOS DE IA MATEMATIZACION DE LA

ECONONIA EN- EI SIGIO XIX

\section{CARLOS CAMIINO MUÑOZ}

(Departamento de Estructura Económica y Desarrollo)

EDUARDO FERNANDEZ BOLLO

(Ecole Normale Supérieure - Paris)

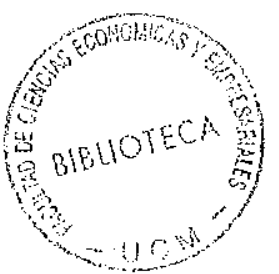

Documento de Trabajo

8605

ORIGENES Y PROBLEMAS TEORICOS

DE LA MATEMATIZACION DE LA

ECONOMIA EN EL STGLO XIX

Carlos Camino Muñoz

Eduardo Fernández Boil 
El espectacular auge de la Economía matemática, que no se ha desmentido desde las postrimerías de la primera gue rra mundial, impulsa, incluso a aquellos que mantienen serias reticencias sobre el tema, a plantearse el problema epistemoIógico de la relación entre las matemáticas (o la Matemática) y la Aconomía. Se plantea, así, la cuestión de saber hasta que punto la matematización transforma no sólo los métodos si no la neturaleza y el estatus epistemológico de la Teoría Eco nomica, ya sea en sentido positivo permitiénciole alcanzar un rigor científico, ya sea de manera negativa haciéndole correr el peligro de desvirtuar su desarrollo propio. Así, durante el Congreso de Economistas de Lengua Prancesa, reunido en Lau sanne para el centenario de Walras, Maurice Allais pudo decir que "Ios trabajos efectuados desde hace veinte años por los economistas han hecho, indudablemente, progresar las matemáticas, pero no la Economía Política" (1)

Sin embargo, al abordar estas cuestiones, nos encon-tramos con un problema preliminar: la complejización y refinamiento extremos del aparato matemático actualnente utilizado provocan la concentración de los esfuerzos de los economis tas en el aprendizaje $\mathrm{y}$ dominio técnico de este aparato $\mathrm{y}$, por Io tanto, se tiende a relegar la reflexión sobre sus condiciones teóricas de utilización. La restricción del tratamiento de este tema solamente al siglo XIX tiene dos ventajas:

- Al ser el instmmento matemático relativamente ele mental, se puede observar con más facilidad como se procede para introducirlo.

- Al ser este el momento del surgimiento de la matematización de la Economía, hay una aguda conciencia epistemológica del problema: los economistas se plantean explicitamente el problema de esa introducción $y$ de su legitimidad.

Así, pues, examinaremos: 10) HI Oxigen de esta matematización, como empezó. $2^{2}$ La Naturaleza de esta ma 
tematización, como se pensó. 3 Las Críticas, que se le for mularon.

\section{IȮ EI ORIGEN DE LA MAATEMATIZACION.}

Como aclaración preliminar hay que precisar que se identificará durante todo este trabajo la primera matenatización de la Economía con lo que se ha venido en Ilamar "Ia revolución marsinalista de los años 1870-80", con la importante salvedad de Antoine-Augustin Coumot, del que ha,remos mención más adelante. Por ello, debemos eliminar dos objeciones a esta identificación que podrían formularse asi: Ya hubo otras matematizaciones previas y no todos los marginalistas son partidarios de la matematización.

A la primera objeción se puede responder facilmente se ñalando que las otras matematizaciones fueron superficiales, es decir que se limitaban a exponer bajo forma matemática cier tas relaciones, pero no convirtieron las matemáticas en un ole mento dinámico del razonamiento económico. Al no aportar nada substancial, estos intentos fueron historicamente inproductitivos (2).

A Ia segunda objeción se ha de conceder que, efectivamente, no todos los marginalistas desarrollan por igual la hatematización. La Ilamada Escuela Psicológica Austriaca mantie ne reticencias al respecto (3). Hay que recordar que a finaIes del siglo XIX la calificación de Escuela hatenática se apl caba unicamente a la Escuela de Lausana fundada por Walras y desarrollada por Pareto. A pesar de todo, si se define al mar ginalismo como un intento de construir la Teoría Económica a partir de la Teoría subjetiva del valor $y$, sobre todo, de la formulación de la Ley de Utilidad Marginal Decreciente, como e sa ley es esencialmente formulable de manera matemática, todas las teorías marginalistas abren la posibilidad de esta primera matematización, aunque la desarrollen de manera desigual.

(2) N. F. Canard. "Frincipes d'Economie Politique. Paris Buisson. 1801.

W. Wervell. Mathematical exposition of some doctrines of political economy". - Cambridge Philosophical Transac tion, vol.3. 1829. vol.4.

(3) C. Pienger "Grundsätze der Volkswirtschaft Iehre".1871.

(4) No se hablará aquí del problema complejo de sus condicio hiatónican tr ancigles de nosibilidad
Una vez aclarado este punto, el problema del origen de la Revolución marginalista se divide en dos: el problema histórico de su aparente retraso y el problema teórico de sus condiciones de posibilidad.

\section{1.- El "retraso" del marginalismo.}

Si conservamos la somera caracterización del marginalismo por los dos rasgos previamente mencionados, la "Teoría Subjetiva del valor" y la "Ley de Utilidad Narginal Decrecien te", podemos observar, en efecto, que estas dos características están ya presentes en la segunda mitad del siglo XVIII. La "Teoría Subjetiva del Valor" está ya desarrolleda por gente co mo Condillac y Gallieni (5) y Daniel Bemouilli formuló por primera vez la forma matemática de la "Ley de Utilidad Marginal Decreciente" en su solución al problema de San Petersburgo (6). $Y$, sin embargo, habrá que esperar más de un siglo para que se desarrolle el maroinalismo (7). ¿Que es pues lo que falta?. ¿Que es lo que ocasiona este retraso?. Exarinando la obra de los economistas deI siglo XVIII, partidarios de la Teoría subjetiva del Valor", se puede responder facilmente a esta pregun ta: esta teoría es incapaz de engendrar una teoría de los pre cios determinada. Is decir, se constmye un modelo teórico de precios que los representa como resultado de las comparacio nes de las utilidades de los agentes del intercambio, pero ese modelo no permite determinar rigurosamente los precios porque hace depender la demanda de un Bien, no de los pre-cios sino de la "necesidad humana", que se define en términos más o menos biológicos. Así, la demanda es un dato independiente del sistema de relaciones de intercambio $y$, por lo tanto, no se puede determinar económicamente, lo que implica la indeterminación del precio. Lo que interesa resaltar episte mológicamente es que no se aplica la "Ley de la Utilidad Decreciente" porque no existe una teoría natenatizable de la de manda: la demanda no es considerada como una variable económica sino que depende de fenómenos biológicos. Por eso la Re volución marginalista no es concebible antes de que Coumot.

(5) F. Gallieni. "Della rionetta". 1751.

Condillac. "Le Commerce et le Gouvernement". 1776.

(6) D. Bernouilli. "Specimen theoriae Novae de finensura Sortis, in "Comentarii Academiae Scientarum Imperialis Petro
politanae". 1733.

(7) Para una exposición detallada del problema ver: 
en sus "Investigaciones sobre Ios Principios Matemáticos de la Teoría de las Riquezas" (8), publicadas en 1838, haga precisamente eso: transformar la Demanda en una varabble intraéconómica, determinada unicamente por los precios, formulando así la Ley de la Demanda $D=F(p)$, la Función de la Renta $D=F$. (D) y Ia función de la Renta margi$\operatorname{nal} \frac{d R}{d P}=F(p)+p F^{\prime}(p)$. (Donde p $=p r e c i o$ de un Bien. $D=$ deman da solvente de ese Bien. $F^{\prime}(p)=\frac{d F}{d p}$.).

Aunque Coumot no comparta la teoría subjetiva del va Ior, su curva de demanda va a dar pie a una teoría matemática basada en esa concepción del Valor. En efecto, Walras se dará cuenta de que la curva de Coumot y la curva de la Ley de Utilidad Marginal Decreciente tienen la misma forma $\mathrm{y}$, por tanto, a la imprecisa formulación seguín la cual "los precios son resultado de la comparación de utilidades" podrá substituir la fómula matemática: $\frac{\mathrm{UX}}{\mathrm{UY}}=\frac{\mathrm{Px}}{\mathrm{Py}} \mathrm{0}$, parafraseando, que el cociente de las Utilidades es igual al cociente de los Precios (9).

Observamos, por ello, sobre este ejemplo, que el desplazamiento conceptual que acompaña a la matematización de la economía es la reducción de una idea más o menos vaga de la Demanda o de la Utilidad a un concepto operativo, es decir susceptiole de figurar en una relación matemática y definido gracias a esa figuración.

II problema teórico que debemos plantearnos a continua ción es el de las condiciones que permiten una serie de desplazamientos de este tipo.

\section{2.- EI paradigma de la matematización.}

La condición decisiva, por lo menos para Cournot, de la matematización de la Economía es la aparición de un nuevo modelo estructural (o paradigma, en términos anglosajones) que hiciese posible e incitase a aplicar las matenáticas a la Economía. Ese paradigma es un paradigma físico (oponiéndose pues a otro paradigma muy corriente y de gran porvenir: el biolósico) pero no un paradigma físico cualquiera sino pre

(8) Sobre Coumot, ver el libro de C. Menard "La Formation "une Rationalité Economique": Coumot. Flammarion, Pa ris 1978. Y su bibliografía selectiva p.311-319. cisamente el que surge con la Mecánica Analítica de Lagrange. La intervención de este paradigma es fehaciente en el Prefacio de las "Investigaciones...." de A. A. Cournot. En efecto, cuando Coumot escribe, la escuela dominante en Fran cia es la Escuela ultraliberal de Paris, que toma como referencia principal la obra de J. B. Say. Y, justamente, J. B. Say ha publicado una crítica violenta de los intentos de matematización de la economía en el Discurso preliminar a la edición de 1826 de su "Tratado de Economía Política". La influen cia de esta crítica será tal que el anatema lanzado contra la Economía Matemática no se resquebrajará en Francia hasta la segunda década del siglo XX, despreciándose la obra de León Walras, que tuvo que emigrar a Suiza para desarrollar sus ideas.

¿En que consiste esa crítica?. Rapidamente se puede ver que Say concibe las matemáticas como unicamente un instru. mento de cálculo numérico, aplicable sólo cuando se pueden es tablecer relaciones numéricas. Como por otra parte sostiene que las cantidades y valores económicos están "sometidos a la influencia de las facultades, necesidades y voluntades de los hombres", se puede saber en que sentido actúan esos factores, pero no se puede apreciar rigurosamente su influencia $y$, en consecuencia, no se pueden establecer determinaciones numéri cas. Las materáticas no sirven, pues, para nada en economía, si se exceptuan las estadísticas, para recoger datos.

A ese ataque frontal, que Coumot no podía ignorar, se encuentra respuesta en el prefacio anteriomente citado. Cour not sostiene que esa crítica viene del desconocimiento de la teoría de funciones (de Iagrange) cuya característica es que permite obtener resultados precisos sin disponer de una evaluación numérica de las relaciones con sólo conocer algunas características de su forma (continuas, decrecientes...). Jus tamente la Mecánica analítica de Lagrange demuestra que se pue den obtener resultados aplicados de este tipo de teoría.

Epistemológicamente, Cournot substituye a la cuestión de. la legitimidad de la matematización la cuestión de la pertinencia del tipo particular de matematización que se ha de utilizar. Es decir, que a una pregunta a priori metafísica en cierta medida (la realidad puede o no ser expresada por la ma temática) y dificilmente decidible, substituye una pregunta a posteriori y que abre posibilidades concretas de discusión y, por lo tanto, de progreso. 
Ia respuesta de Cournot es clara: se puede construir una economía matemática si se expresan las relaciones económicas no como relaciones nuéricas sino como relaciones funcio nìles que posean ciertas características. Pero: ¿Que concep ción de la Economía Natemática sale del desarrollo de essta analogía con irecánica analítica?.

\section{IA NATURALEZA DE IA ECONOMIA MATENATICA.}

No es en Cournot, sino en la obra de Harie Esprit Leon 1 lalras (1834-1910) $\mathrm{y}$, principalmente, en las sucesivas ediciones de los "Ilementos de Economía Política Pura" ( 1 a edición 1874-77; 5ㅇ edisión, definitiva, en 1926) y otros es critos menores del mismo autor donde podemos econtrar no solamente una teoría general, de mucha nayor envergadura que las elaboraciones parciales de Coumot, sino, sobre todo, una conciencia epistemolófica radical y precisa de lo que significa la matematización de la Bconomía. Valras es el primero en afirmar que las matematicas no son un instrumento neutral sino que, para él, cambian la naturaleza de la peoría ticonómica, operan una revolución salileica radical en el terre no de la Iconomía. En ese sentido, su conciencia epistemológica es muy superior a la de un gran matemático $J$ economista de mucha mayor influencia como Alfred harshall, que conserva muchas reticencias respecto a la utilización de las matenáti cas. Evidente muestra de ello es el hecho de que relegue las matemáticas a los apéndices de sus "Principles" (10), cuyo significado es querer creer, aún, que exposiciones en lengua natural y exposiciones en lengua matemática son equivalentes.

Para ver bien el carácter excepcional de la conciencia epistenológica de Halras, se le puede comparar con un economis ta matemático muy posterior: R. G. Douglas Allen. iste exhibe, en su libro "hathematical Economics" (Rac Millan, Iondres. 1959), unos instrumentos matemáticos infinitamente su periores a los de Walras, pero, sin embargo, tiene, todavia, una concepción instrumentalista de las matemáticas. Leemos en el Prefacio de su obra que Allen considera las matemátics cone "un instrumento extremadamente eficiente", capaz de de--

(10) Alfred Narshall: "Principles of Economics", Mac Millan 6 Co. Iondres. I890. sarrollar las consecuencias implícitas en unas premisas (que pueden ser cualquier sistema coherente de axiomas). Como buen anglosajón, Allen advierte que las matemáticas no demues tran, sino que deducen. La demostración de la verdad o ralsedad de unos enunciados sólo depende de la contrastación empírica de los resultados. La diferencia de las matemáticas eco nómicas respecto a las matemáticas puras es que se "viste" al sistema de premisas de un contenido real $y$ eso permite interpretar las consecuencias de inanera real, es decir contrastable.

Es notable que Allen mantiene una concepción logicista de las matemáticas (iInfluído por Hilbert o Russell?) como "Ior ma de razonar" $y$, en eso, entra en total contradicción con la epistemología moderna de las matemáticas que, a partir de Vittgenstein $\mathrm{y}$ Gödel, subraya la diferencia entre la Lósica las matemáticas. Las matemáticas no enuncian tautologías sino propiedades de sistemas de objetos formales. "Objeto formal" debe entenderse en un doble sentido: Primero: un objeto matemático no es un objeto del mundo real. Segundo: es un objeto al que se le imponen ciertas condiciones de forma. In cambio, la Lógica no impone condiciones formales a los objetos de un razonamiento, sino sólo a su forma. Y por eso es por lo que u nicamente puede enunciar tautologías.

Walras, que no pudo sospechar el desarrollo posterior de las concepciones de las matenáticas, es mucho más coherente con la epistemología matenática actual que R. G. Allen. Se da cuenta de que imponer una forma matemática a las premisas no es un acto neutral. Hay que transformar los conceptos para volverlos operativos. Utilizar la Matemática implica redefinir un objeto propio para la Economía, que garantice su au tonomia $\mathrm{y}$ su estatus cientifico. Malras quiere hacer con la Economía la misma transform ción que se operó en Física al a bandonar la Física aristotélica y construir una nueva rísica, autenticamente científica sracias a su matematización.

Pero: ¿cual es ese objeto propio de la Economía Política pura? ¿porqué sólo las matemáticas nos permiten construirlo? Walras nos da en su extensa obra hasta seis tipos de definición diferentes del objeto de la Economía pura. Citaremos aquí sólo. 
una: "El estudio de las leyes necesarias del intercambio, de la producción, de la capitalización y de la circulación en competencia perfecta (11). Si se quiere sintetizar los diver sós aspectos de las definiciones, se puede llegar a una definición desarrollada tal y como la formula schumpeter: "Ql es tudio de un universo cerrado, constituído por el sistema de condiciones o relaciones que determinan los valores de equili brio de todas las variables económicas, los precios de todos los productos y factores y la cantidad de productos y factores que serían comprados en equilibrio perfecto $y$ competencia pura, por todas las empresas y economias domésticas"(12).

Esta definición nos interesa porque postula que el obje to de la economía es un universo cerrado. Universo cerrado quiere decir que tiene dos características. Is completo: to dos los elementos que intervienen están tomados en cuenta. $Y$ es autónomo: todas las relaciones entre los elementos foman un sistema, es decir un conjunto finito de relaciones que per mite determinar, por si solo, todos los elementos que entran en su composición. Una consecuencia protocolaria de este ras go es que no hace falta formular la cláusula "ceteris paribus" para todos los elementos y procesos no económicos. Sus varia ciones no tienen importancia, no determinan ningrín valor ecom nómico. Pero ino es eso arbitrario?, icomo podemos estar seguros de que el objeto de la economía es un universo cerrado?. Justamente, para Walras, son las matenáttcas las que permiten justificar este postulado. En efecto, si el sistema de todas las relaciones económicas es expresable en un siste ma de ecuaciones, entonces, si se dan ciertas condiciones per fectamente verificables (que el sistema sea consistente, com porte ecuaciones independientes no reducibles a identidades $\mathrm{y}$ en número igual al de las variables), se puede afirmar sin nin gún dogmatismo que este sistema determina un universo autónomo. Las matemáticas son, pues, condición necesaria y suficiente para afirmar a la Economía como ciencia PURA: sin las matemáticas, el postulado de un universo cerrado es arbitrario $e$ inverificable.

Por eso es esencial que la economía científica formule to dos sus conceptos de manera que sean matemáticamente operati-

(II) "Discurso del Jubileo" vos. Todos los elementos económicos deben ser considerados como magnitudes $\mathrm{y}$ todas las relaciones económicas como funciones de ciertas características. El problema de Walras, pionero de la matematización, es que, para que sus matemáticás puedan aplicarse, hacen falta aún muchas más condiciones restrictivas, que Schumpeter califica de simplicaciones heroi cas. Examinaremos una de ellas para ver que justificación es capaz de aportar Valras.

Walras supone y construye como hipótesis explicativa la idea de un equilibrio perfecto, es decir, un equilibrio en el que las fuerzas que 10 establecen, cuando son ligeramente modificadas, provocan reacciones en sentido contrario que tienden a restablecer el estado primitivo. Como las fuerzas eco nómicas son para Walras el comportaniento de los agentes eco nómicos (empresas, economías domésticas) individuales, ese re quisito se traduce por la idea de que los agentes económicos se comporten como un "homo oeconomicus". Este es un agente que quiere y puede (dándose ciertas condiciones como una información perfecta) comportarse de una manera totalmente ra,mon cional, es decir maximizando la utilidad total que puede alcanzar con los recursos de que dispone.

Lo Interesante es darse cuenta de que la justificación del "homo oe conomicus" no es principalmente psicolófica, sino metodológica. Walras no saca esta hipótesis de un estudio psi cológico positivo $\mathrm{y}$, desde ese punto de vista, su hipótesis es una simplificación abusiva. Pero, metodológicamente hablando, su hipótesis es la condición de abstracción sobre los agentes económicos que permite matematizar sus relaciones. por lo tan. to, Walras podría justificarla de manera mucho más convincente utilizando, una vez más, su continua analogía con la física(13). Del mismo modo que es lícito en Física abstraer del objeto de la experiencia sus cualidades sensibles para construir el objeto abstracto de la Física y sus leyes necesarias, podría ser lícito abstraer ciertos rasgos del agente económico concreto para poder aislar las "leyes necesarias" de las relaciones eoo nómicas.

Resumiendo las conclusiones del análisis de las concepolo nes epistemológicas de Walras, podemos decir que, para él, ma-

(13) Ver, por ejemplo, su conferencia "Economie et Mécanique.

(I2) "History of Economic Analvsis". Parte Tr. Canitulo 7a, 
tematizar la Economía es hacerla acceder a un estatus autenti camente científico, porque:

10 Al permitir formular de manera operativa los concéptos fundamentales imponiendo una serie de condiciones de abstracción sobre la naturaleza de los agentes económicos y de su comportamiento, hace disponer a la Economía de conceptos precisos.

22 Al formular el conjunto de las relaciones económi cas fundamentales como un sistema coherente de ecuaciones, permite constituir un universo cerrado como objeto propio de la Economía.

30 Pone a disposición de la Economía un instrumento de deducción mucho más eficaz que el razonamiento intuitivo $\mathrm{y}$ perfectamente adaptado a su objeto.

Lo interesante de esta concepción es que, independientemente de los supuestos heroicos que integra, es epistemológi camente coherente como programa normativo de la Economía: si se quiere obtener una teoría económica pura hay que utilizar las matemáticas o caer en Io arbitrario. Por otra parte, como mínimo, gracias a la utilización de la analogía física, bas ta para afirmar que no se puede rechazar la matematización a priori sólo porque necesite imponer hipótesis de abstracción. La Física ha demostrado que la abstracción puede ser fecunda para estudiar objetos reales.

Por 10 tanto, este análisis de Nalras nos permite divi dir las críticas de la matematización en tres grupos: las crí ticas obscurantistas, las críticas económicas y las críticas matemáticas.

\section{IAS CRITICAS DE IAA MATEMATIZACION.}

\section{1.- Ias críticas obscurantistas.}

Se puede calificar de tales aquellas críticas que rechazan a priori toda matematización en virtud de un mo delo asumido acríticamente de lo que debe ser la Economía. Co mo ejemplo podríamos tomar el caso anteriormente citado de J. B. Say $y$, sobre todo, de sus discípulos de la Escuela de Paris. Podríamos añadir a esta lista el caso del mate mático J. Bertrand en su análiisis devastador de las tesis de Cournot y Walras, publicado en el "Journal des Savants", en septiembre de 1883 .

\section{2.- Ias críticas económicas.}

Estas críticas se diferencian de las del primer grupo porque, en vez de criticar la matematización a priori y en bloque, parten de unas criticas concretas que permiten señalar límites definidos de esta matematización. Es decir, que consideran la matematización insuficiente para constituir, por si sola, un universo cerrado de fenómenos económicos, por que ser̃alan ciertos factores concretos importantes desde el punto de vista económico que se resisten a la matematización. Un ejemplo podría ser el de Alfred harshall cuando señala los límites de la conceptualización matemática de su tiempo, haciendo resaltar su incapacidad para integrar un análisis diná mico y temporal de los fenómenos económicos. Otro ejemplo es taría constituído por el marxismo. In el famoso capítulo sobre el fetichismo de la mercancía, Marx sostiene que las rela ciones entre cosas son esencialmente relaciones sociales encu biertas por esa forma fetichista de relaciones "objetivas" en tre las cosas. A partir de esta tesis se comprende que las relaciones sociales $y$ sus características (conflicto, crisis.. ...) son hechos económicos determinantes y que, sin embargo, no se dejan matematizar facilmente.

Ia característica da estas críticas es que son a posterio ri y concretas. Son críticas que se hacen a una matematización particular sobre la base de unas concepciones económicas particulares. Por lo tanto no pueden ser consideradas como recusreates; definitivas, puesto que siempre cabe la posibilidad de que sean superadas por construcciones más refinadas y elaboradas. Integrando esas críticas es como, de hecho, se ha realizado el progreso de la Economía lifatemática. Por ejemplo, se han desarrollado construcciones matemáticas dinámicas y has ta métodos matemáticos para pensar relaciones de conflicto en tre individuos o grupos (cf. la teoría de los juegos, de Von Neumann $y$ Miorgensterm)

\section{3.- Las críticas epistemológicas.}

Este tipo de críticas abarca aquellas que intentan. precisar las condiciones generales de atribución de un signifi. 
cado económico a una construcción matemática. Son por lo tan to recurrentes, puesto que se aplican a toda construcción matemática. Sin embargo, por razones obvias, no son críticas a priori que rechacen toda matematización, puesto que sólo intentan precisar sus condiciones de validez.

Un primer ejemplo de estas críticas se encuentra, a mo do de chiste, en la famosa carta de inarshall a Bowley del 27 de febrero de 1906, que nos da una serie de "reglas para el buen uso de las matemáticas". Darshall insiste sobre la necesidad de una "traducción" a téminos reales de los resultados matemáticos. Sin la posibilidad comprobada de esa traducción, el resultado matemático no sirve para nada en Iconomía.

Por su parte, el matemático y epistemólogo francés, Hen ri Poincaré, en su breve correspondencia con Leon Talras, avan zaba criticas de tipo similar. Walras le había enviado un ejemplar de sus "Elementos de Economía Política Fura" para intentar conseguir, gracias al prestigioso matemático, un "espal darazo" que le permitiese romper el aislamiento y. el desconoci miento de su obra en Francia (14). Walras, en la segunda carta que envió al físico, insiste una vez más sobre la analogía entre su labor teórica en el campo de la economía y la construcción de una física matemática. Poincaré le responde con mu--..chas precauciones, admitiendo la posibilidad a priori de una economía matemática (Io que le diferencia de J. Bertrand), pe ro insistiendo sobre las precauciones que hay que tomar para eliminar los elementos arbitrarios de este tipo de constmicción. In efecto, Poincaré estaba, como epistomólogo, muy sensibiliza do sobre el problema de la interpretación del significado no. matemático de fómulas matemáticas. Inspirándose libremente en su obra como epistomólogo de la Fisica podemos precissar tres tipos de precauciones que hay que tomar para eliminar in terpretaciones arbitrarias (15).

A) No hay que confundir el significẻdo económico del con cepto matematicamente operativo con el del concepto intuitivo. del que procede. Es decir: no hay que olvidar las condicior. nes restrictivas que se han impuesto para pasar del uno al of tro. Podemos dar dos ilustraciones de confusiones basadas en el olvido de este precepto: Primero, en lo que concierne a la

(14) "Correspondence of Ieon Walras, edited by A. Jaffe. T. III, $\mathrm{pg}$. $158-165$. teoría del monopolio. En su forma neoclásica, es una teoría de un vendedor único que no puede influir sobre la curva de demanda. Esta teoría, evidentemente, no corresponde a muchas rélaciones reales donde el vendedor único si puede influir so bre la curva de demanda. Sin embargo, hasta la elaboración por P. Sweezy de la curva de demanda quebrada en su famoso ar tículo de 1937, los economistas hicieron como si se aplicase a todos los vendedores únicos. Un segundo ejemplo sería la confusión que hace Walras entre su teoría estática del equilibrio y una teoría de un estado de equilibrio estático (o es tado estacionario). Walras confunde una ficción metodológica, que construye un concepto de equilibrio que no hace intervenir el factor tiempo, con la descripción de un hecho real, a saber, un estado en el cual el tiempo no produce ninguna va riación.

B) No hay que confundir una ficción metodológica con una constmicción teóricamente normativa. Is decir, no se deben considerar las ventajas formales de un concepto como ven tajas de significado económico. Así, el concepto de competencia perfecta posee muchas ventajas formales puesto que per inite construir, en el marco de ciertas hipótesis de abstracción, un sistema de equilibrio perfectanente determinado. Pero ello no quiere decir que todo análisis de los fenómenos económicos tenga que tomar como punto de partida esas hipótesis de abstracción. Sin embargo, muy posteriomente a Valras, Hicks, en su libro "Value and Capital" (1939), piensa aún que "abandonar la hipótesis competitiva hace correr el ries go de arminar la mayor parte de la teoría económica". Hi gue decir tiene que es aún mucho más arbitrario considerar la ficción metodolófica de la competencia perfecta como una cons. trucción politicamente normativa.

C) Al construir una teoría no debe guiarse la elaboración por criterios formales (simetría de los conceptos opera tivos, posibilidad de simplificar formalmente la construcción), dejando de lado los critexios de pertinencia económica. Un ejemplo de este tipo de error se encuentra en la teoría margi-. nalista de la producción. Como señala Pasinetti en su libro "Lecciones sobre la teoría de la producción", los marginalis tas intentaron aplicar su modelo fundamental, que era un mode Io de intercambio puro, al análisis de los procesos de producción. Para ello intentaron pensar el proceso de produc- 
ción con categorlas análogas formalrnente con las que utilizą ban en su modelo de intercambio, sin plantearse el problema previo de su pertinencia en otro terreno de análisis. Asi laboran la idea de productividad marginal por analogía con la de utilidad marginal, la idea de substitución entre diversos factores de producción por analogía con la de substitución en tre diversos bienes de consumo.

¿Que conclusiones podemos sacar del examen de estos tres tipos de críticas?.

La primera, de tipo general, es que no existen limites teóricos a priori de la matematización, sino precauciones a priori que hay que observar para precisar la validez y el sig nificado económico de un razonamiento matematizado $\mathrm{y}$, por otra parte, limites a posteriori y concretos que hacen surgir las tras teorías económicas. Pero de la existencia de estas precauciones a priori y de estos límites concretos se pueden sacar otras consecuencias importantes.

10. Que existen criterios epistemológicos de preferencia entre las diversas construcciones matemáticas ( $y$ no mate máticas). Por ejemplo, en el caso de las teorías de producción previamente mencionadas, existe una preferencia epistemológica hacia aquellas que intentan construir sus conceptos guiándose por un análisis de los procesos económicos y no por paralelis mos formales. Así la teoría neoclásica de la producción se presenta como una extensión arbitraria de una teoría que se o cupa de la asignación óptima de ciertos stocks de recursos a un terreno económico que, sin embargo, parece estar concemido mucho más por magnitudes flujo que por magnitudes fondo. Ia preferencia epistemológica va a aquellas teorías como las que, a partir de Sraffa y Pasinetti, se intentan consiruir con matemáticas mas centradas sobre los aspectos propios del proce so de producción.

20. Que, dentro del marco de las precauciones ya mencionadas, la utilización de las matemáticas sirve, efectivamen te, para clarificar y precisar los problemas económicos y que, incluso, las perspectivas que rechazan'la construcción de una teoría económica pura no pueden ignorar las ventajas de una formulación matemática, siempre que sea posible dotarla de un significado económico. Epistemológicamente esto quiere decir que el campo de la economía matemática es mucho más amplio que el de Ia teoría económica pura. I todas las objeciones concretas que se le hagan pueden ser consideradas como los agen tes del progreso ulterior de las construcciones matemáticas. Un ejemplo de discusiones que, dentro del rnarco de una teorí no fundamentalmente matemática, han ganado en precisión gracias a una mayor elaboración maternática es el problema clave para las discusiones entre el marxismo y las corrientes neoricardianas, de la transformación de valores en precios, sobre todo a partir de las contribuciones de Tugan-Baranowsky y I. Bortkiewicz (16).

30. Sin embargo, el problema epistemológico de plalras de constmir una teoría económica pura grscias a las matemáticas, aunque sea coherente, está permanentemente subvertido por 1a. necesidad de hacer intervenir nuevos factores. Is decir que el hecho de que la frontera entre factores económicament relevantes $\mathrm{y}$ factores económicamente no pertinentes sea ines table, hace muy aleatorio el horizonte walrasiano de completud del objeto de la Economía.

A partir de esta conclusión se plantea la siguiente alter nativa epistemológica: Si se quiere mantener la idea de una teoría económica pura, que enuncie leyes necesarias y universa les, se debe seguir siendo epistemológicamente walrasiano (Io que no quiere decir quedarse encerrado en la teoría decimonónica concreta de I. Walras), pues sólo las matenaticas garanti zam que se pueda enunciar leyes de este tipo. Pero, entonces, hay que asumir el hecho de que el objeto de esa teoría es una ficción metodológica, cuya validez está supeditada a las posibilidades de utilización que permiten sus condiciones restrictivas matenáticas. El otro término de la altermativa es soste ner que la Economía se ocupa prinordialmente de unos procesos históricos, entre los cuales es arbitrario decir que rigen leJes necesarias y universales. Tan solo se pueden circunscribir "modos de funcionamiento" (Althusser), de los cuales sólo unos segmentos parciales son matematizables. Eso no Implica que la Economía, corno ciencia autónoma, no exista, stno que es tá integrada en una teoría general de los procesos históricos. Esta actitud epistemológicamente corresponde bastante bien a la del materialismo histórico. El problema de esta actitudes

(16) M. Tugan-Baranowsky, "Theoretische Grundlacen der ilarkis mus". Leipzig 1905

I. Von Bortkiewicz (Traducción froncesa del original ale 
que debe renunciar a la precisión de las matemáticas para una teoría general de los fenómenos económicos y debe, pues, formular criterios de pertinencia de las explicaciones econó micas suficientemente precisos para que permitan discriminar los. 